\title{
A Comparative Study on the Spatial Consciousness of Traditional Paintings in the East and the West
}

\author{
Xiaoke Yin* \\ Weinan Normal University, Weinan 714000, Shaanxi Province, China \\ *Corresponding author: Xiaoke Yin, dafei8287@163.com
}

\begin{abstract}
This article compares and analyzes the development history, ideological culture, and philosophical concepts of traditional paintings, landscape paintings, and still life paintings in the East and the West. The essence of painting is a form of visual consciousness. There is a unique way of processing and expressing spatial consciousness in different images, regions, and humanistic spirits of Eastern and Western paintings. The difference in spatial awareness promotes mutual learning, guidance, and promotion between the Chinese and Western art which have different historical backgrounds, aesthetic concepts, and national customs. Therefore, different ways of paintings would also have differences in the spatial consciousness of the paintings.
\end{abstract}

Keywords: Aesthetic ideas; Perspective; Eastern and Western painting; Spatial consciousness

Publication date: July 2021; Online publication: July 30, 2021

\section{Introduction}

The essence of painting is a form of visual consciousness. Different nationalities, cultures, economies, and historical backgrounds will have different spatial awareness in paintings. Different pictures have different spatial structures and expression techniques. Chinese traditional painting with writing brush, ink, mineral pigments, silk, and Xuan paper as tools (raw materials) can be divided into three forms which are the fine brushwork, freehand brushwork paintings, and the concurrent form. In contrast, Western paintings have unique painting languages such as line, light, shade, volume, and color. There is a unique composition and a unique way of expression in paintings. The two are not mutually exclusive but they are unified. This article carries out a comparative study on the spatial awareness of traditional Chinese and Western paintings in order to make a preliminary discussion.

\section{Influence of painting concepts and aesthetic concepts on spatial consciousness}

Western paintings use perspectives and optics to draw things very realistically. In ancient Greece and Rome, decoration has been the main purpose of painting. While paintings express beauty, they also express truth. An example of a Western painting is shown in Figure 1.

The artistic views and creation of Chinese paintings require painters to carefully observe nature and portray nature more faithfully. The objectivity and subjectivity of Chinese paintings are the content of ideas, a form of consciousness, spirit, and condensed feelings which have expressive and reappearance factors.

The artistic views of Western paintings require painters to be faithful to objective objects, to grasp the reality of the world, and to pay attention to the harmony of things in the universe which have strong impacts. The painters use the scientific method of perspective, light, color, etc. to paint real things that they see. This embodies the expressive techniques of Western paintings. Moreover, the use of one-point perspective or 
focal perspective in traditional Western paintings has become a major feature.

On the one hand, Western classical paintings express the reality of the world, but on the other hand, they express the harmony of space. Their paintings have a strong visual impact, strict scientificity, and harmonious rational thinking. In Hegel's summary of painting, he stated, "Each style aims at depicting nature faithfully, and nothing else, but each has a different concept of nature." Three-dimensional angles illustrate the relationship between color brightness, purity, and hue. Through the analysis of spectral tones, there is a color relationship, a color circle, the relationship between color and light, as well as the relationship between color and vision. The shadow of the object is the complementary color of the light source, and the external light sketching draws the shadow of the scene into vivid colors. Note that the rational attitude of rewriting reality creates the pursuit of true spatial depth and infinite spatial awareness in art.

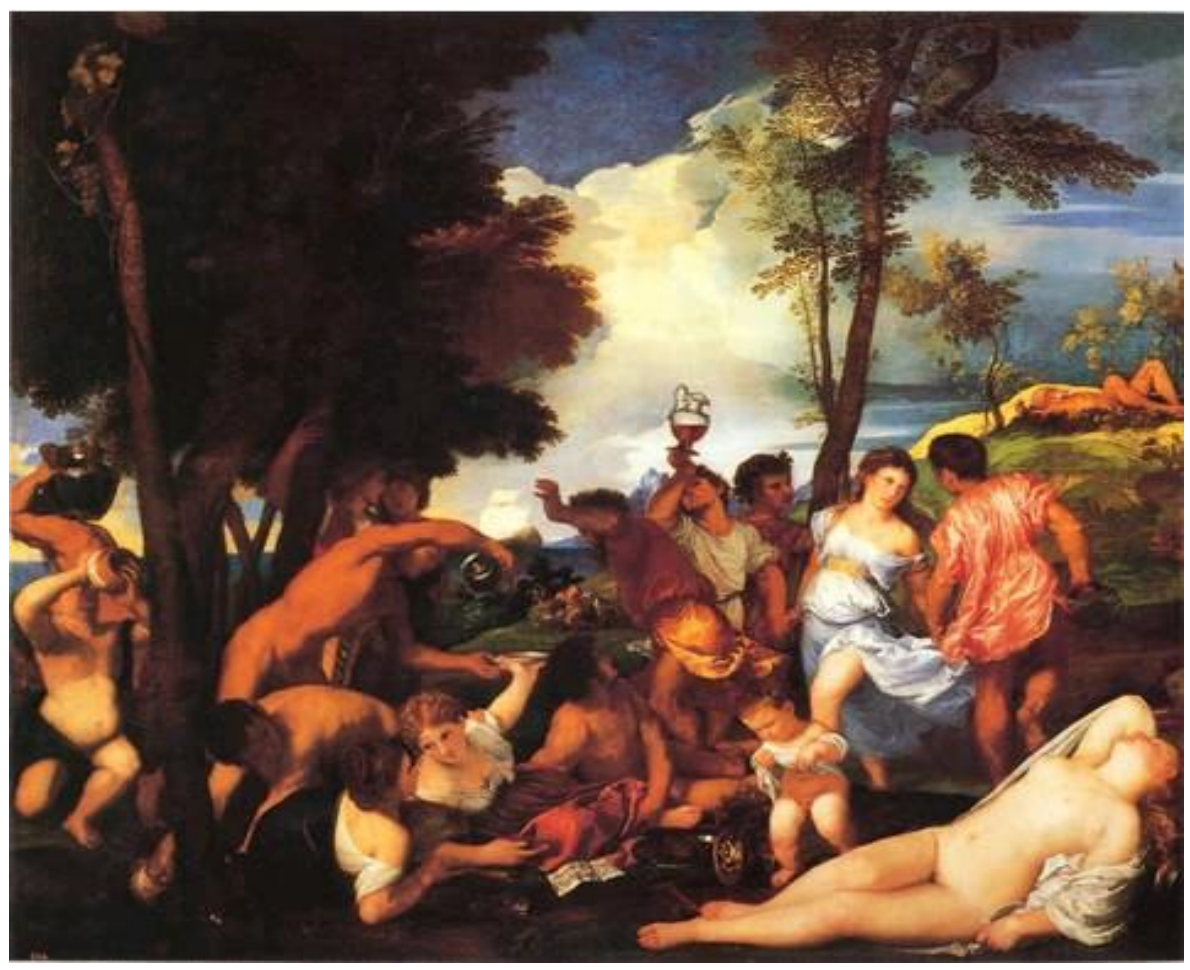

Figure 1. Bacchanal (1523-1524) by Tiziano Vecellio

Chinese paintings emphasize artistic conception rather than form. The artistic conception exists in the image, and it is also expressed in the brush and ink technique. The brush and ink technique naturally affects the subjective expression technique. The main purpose of the creation is in the brush and ink where both the brush and ink have the artistic function of a lyrical object which allows the painting to demonstrate the charm of the brush. Chinese paintings break a series of rules of time and space, drawing everything in the picture. The scattered perspective of Chinese landscape paintings combines time and space.

“A Panorama of Rivers and Mountains" (千里江山图卷) by Wang Ximeng (Figure 2) who is a painter from the Northern Song Dynasty used the scattered perspective of Chinese landscapes which gives full play to the advantages of the special perspective of Chinese paintings so that the admirer has a limit that goes beyond the point of view. As if looking down from the sky, the panoramic view of the earth stretches with the ups and downs of the mountains and thousands of miles of rivers. The selection of these elements expounds more about the laws of the universe, the roots of all things, as well as people's expectations and 
yearning for beautiful things. It reflects people's ability to perceive everything in the world in which it is more of the formation of concepts and consciousness. These concepts and consciousness have promoted the prosperity and development of social culture, bringing gorgeous images that shock and inspire souls through people's visual aesthetics. There are countless works of scattered perspective in which Zhang Zeduan's “Riverside Scene at Qingming Festival; 清明上河图” from the Song Dynasty is an excellent work that fully reflects the scattered perspective.

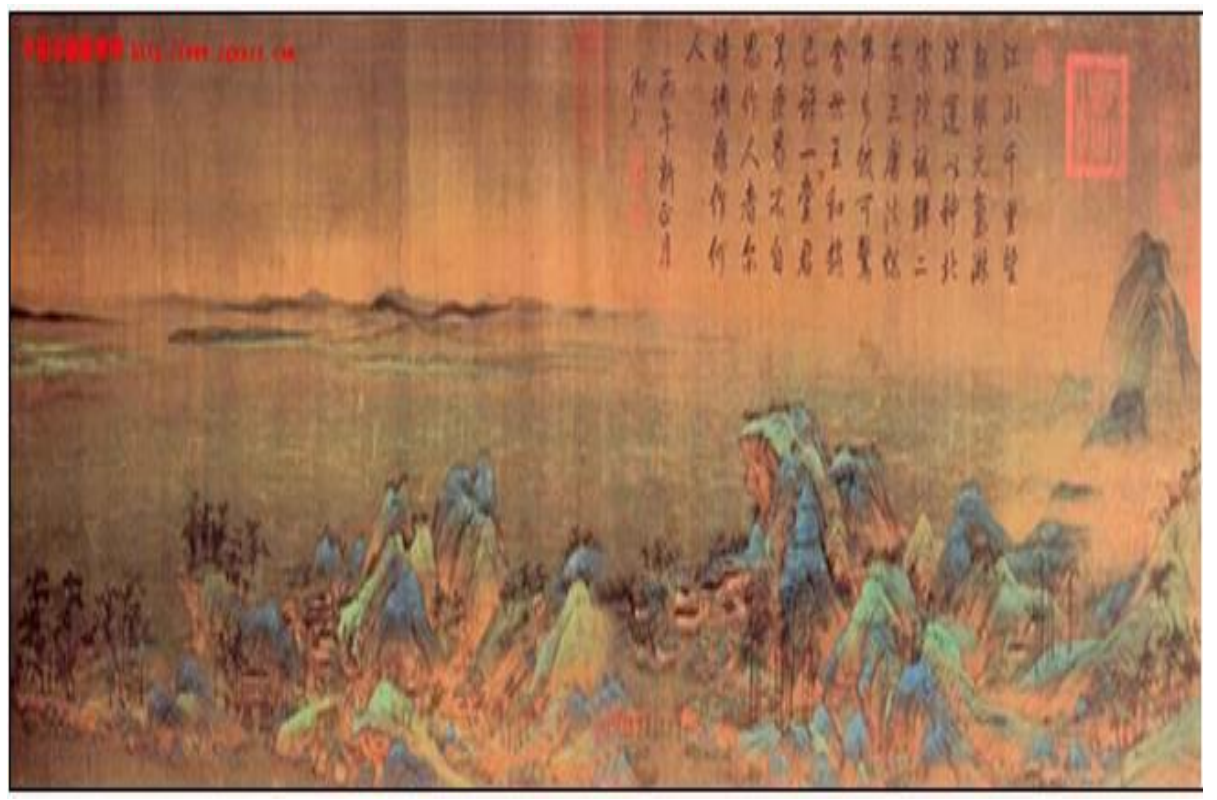

Figure 2. A Panorama of Rivers and Mountains by Wang Ximeng from the Northern Song Dynasty

There are many works of traditional Chinese paintings that embodied emotions in sceneries such as Shen Zhou's works; “The Delightful Cangzhou Landscape,” (沧州趣图) and “Appreciating Potted Chrysanthemum in Tranquility” (盆菊幽赏图). This habit of using paintings to express emotions and thoughts has spread to the modern society along with social development. The landscape painting style in Shen Zhou's works that borrows sceneries to express emotions and empathize with the world has also become a major feature. The landscape painting style and its other-worldly ideas have been passed on ${ }^{[1]}$. "The spirit and rhyme are vivid; the brush is used in the bone method." The bone method uses the brush where the brush is strong, rich, and simple. One can experience the state of writing in paintings. The breaking of the glyph structure in Chinese characters and the rearrangement of the paintings are observed in order to obtain a new visual aesthetic effect from the ever-changing interspersed ink lines. "Margin leaving" in landscape paintings has always been concerned as a vital part of the black and white system of Chinese paintings. It uses limited pictures to express infinite space. Expressing emotions are characteristics of Chinese paintings.

For example, Shen Zhou's landscape paintings use aspirations, emotions, and artistic conceptions. The abstraction of lines brings strong appeal and infinite vitality to the painting. The texture and strength of the lines make the work itself expressive. It is powerful in showing the temperament and spiritual realm of literati painters where the culture of the Ming Dynasty is a sign of maturity. In the painting, the unique art form of pen and ink as well as the Zen-like pen and ink technique show that people's feelings and moods are often the same in a certain era. It is also of great significance to study art works from an aesthetic perspective ${ }^{[2]}$. 


\section{Spatial representation of Western painting}

The spatial representation of Western paintings is the result of the scientific use of perspective. In da Vinci's opinion, a picture is like a mirror which must present a real three-dimensional scene in a two-dimensional plane. Like Repin's "Barge Haulers at Volga," scientific methods such as perspective, light, and color have been used to paint the real things that are seen. This embodies a technique of Western paintings. The first is the focal perspective method where the accurate perspective relationship on the screen is an important feature of the spatial processing in Western paintings. Lessing, the $18^{\text {th }}$ century literary theorist said, "Only visual experience, making the far things smaller than the near ones is far from enough to constitute the perspective of a painting. Perspective must have a specific point of view and a clear view of nature; these are what ancient paintings did not have." Observing the shape of objective things is mainly based on vision and the shape of various things can show the shape of the object only with the help of light. Comparing "The Gleaners" by Miller (Figure 3) and "The Avenue at Middelharnis" by Hobbema (Figure 4), the former depicts more spatial levels. Placing the horizon line very low, it can be appreciated that the position of the artist is also very low and the strong one-point perspective of "The Path in the Woods" has strong visual impact.

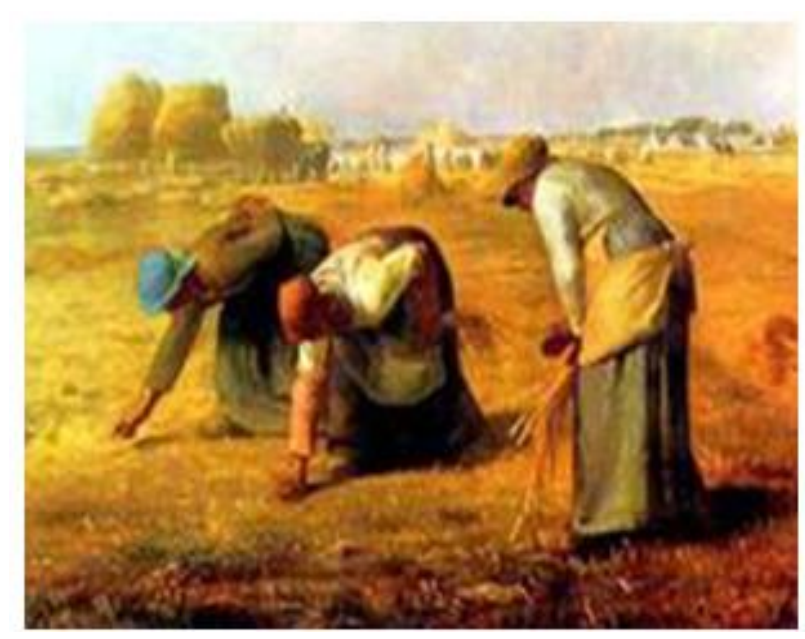

Figure 3. The Gleaners by Miller

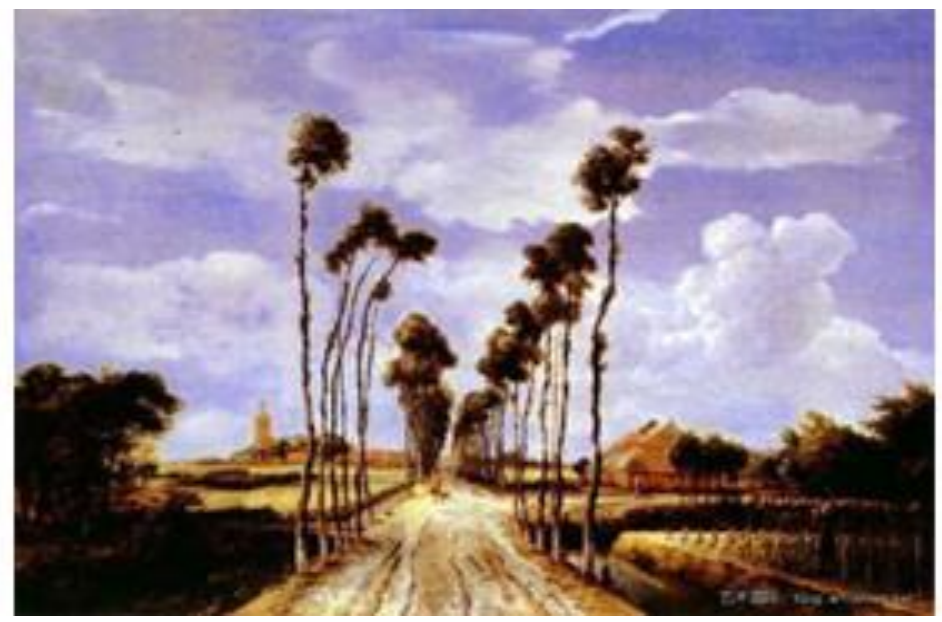

Figure 4. The Avenue at Middelharnis by Hobbema

The second is the perspective method of light and shade which is also commonly referred as the method of light and shade. This method uses the technique of presenting a three-dimensional effect of an object on a two-dimensional plane. The chiaroscuro method came to a mature stage in the heyday of the Renaissance. Leonardo believes that objects in the objective world use light and shade to present images; hence, paintings should also focus on expressing light and shade rather than depicting contours. Seek balance in contrast, and contrast in balance. In the shaping stage, shadows are important in chiaroscuro because if there are no shadows, the boundaries of opaque three-dimensional objects would be unclear. The coordinated relationship formed by the changes in the overall painting, the strength and distance from the light source, the decent turning of the object, and factors such as forward light, backlight, ambient light, high light, and bottom light would change the texture of the object.

The third is aerial perspective. The lines used are different depending on the distance of the object. The rule is that the near object is clear, thick, and long while the far object is blurred, thin, and short. These are the characteristics of aerial perspective. In Michelangelo's “The Last Judgment” (Figure 5), the blue sky is the background, highlighting the main body to give people a strong visual impact, reflecting bright colors. This indicates that the aerial perspective in Western paintings also shows a kind of illusory reality that completely reproduces the real space. 


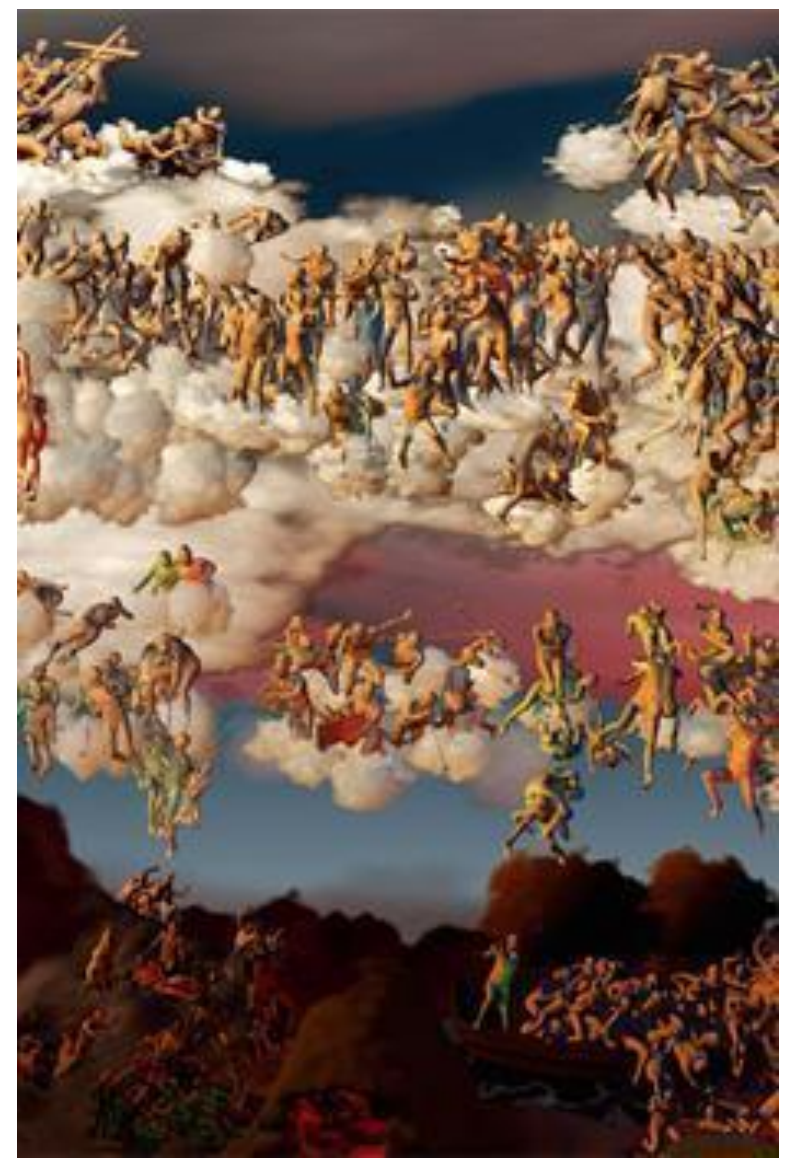

Figure 5. The Last Judgement by Michelangelo

\section{Spatial representation of Chinese painting}

The spatial representation in Chinese paintings is different from Western paintings that adopt the principle of proportion and optics but uses an abstract brush and ink to express it.

First of all, the expression of spatial consciousness in Chinese paintings relies on calligraphy. It is a unique art form in China with a long history and can best reflect the spatial ideology of Chinese people. Each Chinese character expresses a certain space. As Chinese characters are composed of various strokes, there is a blank space between the strokes.

Secondly, Chinese landscape paintings are independent forms of paintings. They are symbols of Chinese people's integration with nature. The space in the paintings has differences in the distance between the front and the back but without changes in the near and far which reflects the unique way of thinking and humanistic spirit of the Chinese people. Yang Yongliang's “Evening Song of Qiushan” (秋山唱晚) (Figure 6) retained the burnt ink painting method, comprehensively used water and ink, ink and color, and other techniques to present a beautiful aesthetic appeal in achieving the appreciation of elegance and commonness of art.

Zhang Zao has put forward the idea of "Teaching fortune, but also gaining the heart." Shi Tao emphasized that "one painting" and "painting quotations" are to break the boundaries between philosophy and art. The spirit of Tao in China originated from the understanding and thinking of the universe. The artist seeks a fitting point in his artistic creation to express the natural beauty and personal perception.

The Northern Song Dynasty landscape painter, Fan Kuan painted "Travelling amid Streams and Mountains" (溪山行旅图) (Figure 7) where he used a lofty panoramic composition to fully express the majestic momentum of the northern landscape. The mountains and rocks in the painting show the vigorous charm of the mountains and rocks with dense ink marks and jagged rock textures. 


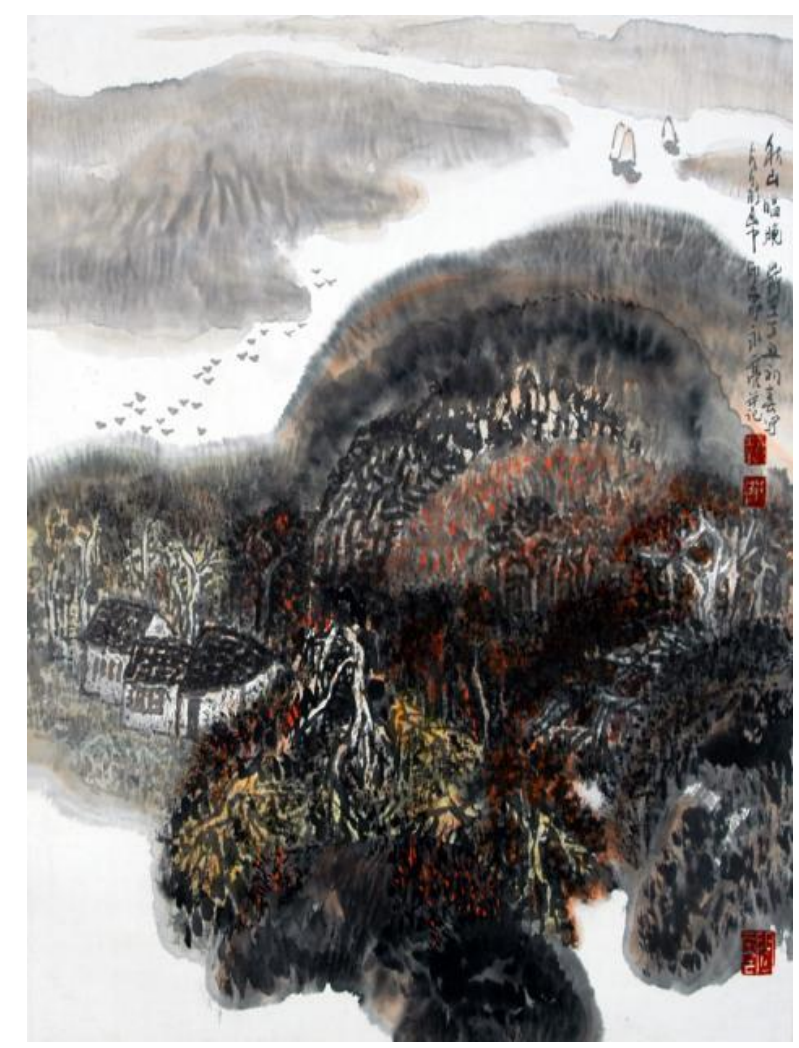

Figure 6. Evening Song of Qiushan by Wang Yongliang

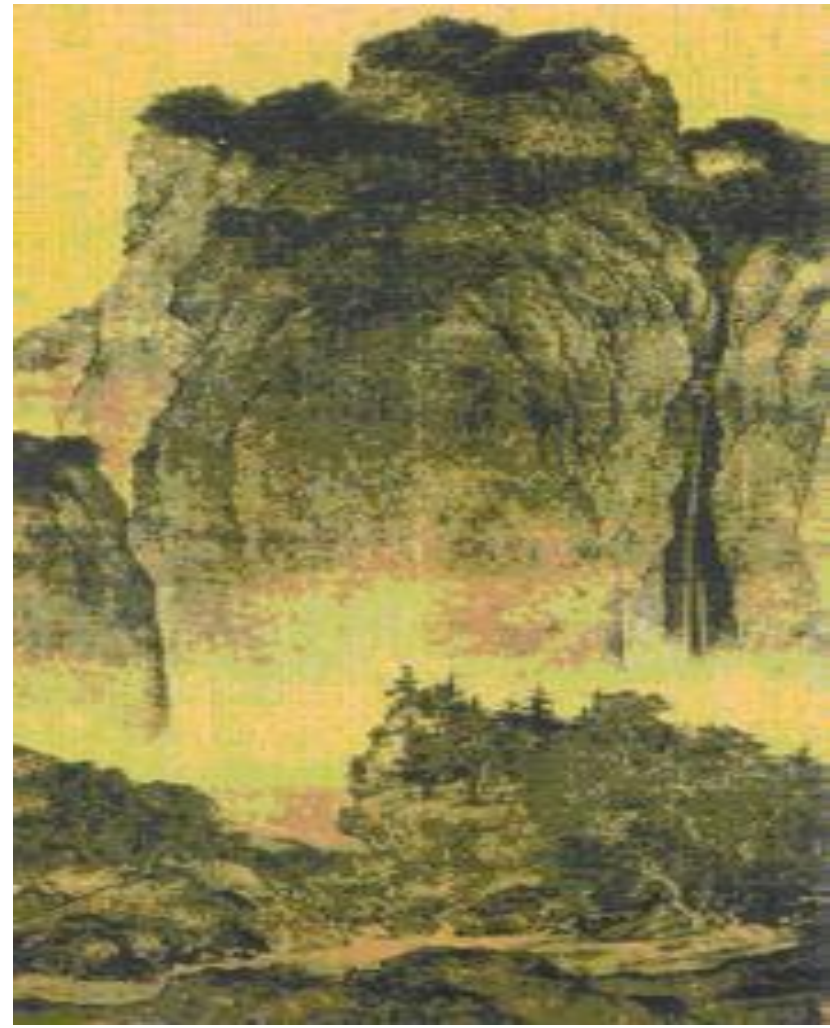

Figure 7. Traveling amid Streams and Mountains by Fan Kuan

\section{Conclusion}

In short, the scattered perspective is unique to Chinese landscape paintings, and it reflects the basic requirements and characteristics of Chinese paintings. Western paintings emphasize on focal perspective, color perspective, light and dark perspective, as well as parallel perspective to pursue three-dimensional space in addition to strictly following the principle of scientific perspective to truly reproduce the image and in making the picture feel authentic. Chinese paintings reflect a Chinese-style outlook on life, close to the essence of art. There is such a saying, "Chinese painting is the science of art, and the Western art is the science of science." Through the comparative study of Chinese and Western paintings, clarifying the respective development trajectories in the paintings of the two systems points out the direction for the further development of art paintings in China.

\section{Disclosure statement}

The author declares that there is no conflict of interest.

\section{References}

[1] Cheng C, 2005, Sketch, Sichuan Fine Arts Publishing House, Chengdu, 8-9.

[2] Ma Y, 2001, Painting techniques, Southwest Normal University Press, Chongqing, 7-8.

[3] Ye Z, 2012, A visual analysis of Chinese and Western painting methods in space. Literary Controversy, (7): 8-9.

[4] Zhou R, 2009, Research on the differences in spatial consciousness of Chinese and Western paintings. Fine Arts Grand View, (7): 4-7. 